\title{
Study on the fractal model of water immersed collapse of Soft Rock
}

\author{
Lujun Ding (Main and Corresponding Author) \\ Department of Civil Engineering, Sichuan College of Architectural Technology \\ 618000, Deyang (China) \\ mddh966@126.com \\ https://orcid.org/0000-0003-3472-3154
}

\section{Yuhong Liu}

Department of Civil Engineering, Sichuan College of Architectural Technology

618000, Deyang (China)

mddh9666@hotmail.com

https://orcid.org/0000-0003-1382-2268

Manuscript Code: 13894

Date of Acceptance/Reception: 05.11.2020/13.05.2020

DOI: 10.7764/RDLC.19.3.486

\begin{abstract}
Soft rock is a common rock mass in engineering, one of its characteristics is water swelling and disintegration. In this paper, the nonlinear fractal geometry is introduced and the correlation fractal dimension is used to study the characteristics of slate disintegration, based on the laboratory test of water immersion disintegration, the method of quality fractal dimension is used to solve the fractal dimension of the disintegration of slate, and the change of fractal dimension is used to reflect the characteristics of the softening and disintegration of slate when encountering water. The experimental results show that the fractal model can be used to fully understand the development and evolution of rock disintegration process, and to quantitatively link the relationship between rock expansion and disintegration. The conclusion has guiding significance for engineering practice.
\end{abstract}

Keywords: Slate, disintegration, drying-wetting cycle, fractal dimension, scrap

\section{Introduction}

The disintegration of rock refers to the property that the rock loses its cohesiveness when it interacts with water and becomes a loose material completely losing its strength. The collapse of rock can cause the slope to peel off, form rock cavity and induce collapse, which provides material source for landslide, debris flow and other disasters, and is the cause of many kinds of geological disasters(Tating Hack and Jetten, 2013). Slate is a common rock mass in engineering. Because of its low strength and poor cementation, it is easy to expand and disintegrate when encountering water, therefore, the research on the disintegration of slate has important theoretical and practical significance.

Erguler (2009) defined the disintegration ratio as the area under the grain size distribution curve of the disintegrated material to the total area encompassing all grain size distribution curves of the samples, was used to account for fragmentation into varying sizes. Zhu, Liu \& Lv, (2017) studied the factors that affect the failure mechanism of rock based on discrete element method. Through performing direct shear tests on different sized replicas cast from various natural joint surfaces, Bandis Lumsden \& Barton(1981) studied the effect of scale on the shear behaviour of joints. Vtorushin (2019) presented a new dynamic model enables one to predict the failure zones. It has been shown that the initial shear stress at the cutter tip causes the disintegration developing deep into the medium and along the cutting direction with contouring of the rock failure scale. Lin \& Chien (2017) proposed a structural health monitoring system based on multifractal detrended cross-correlation analysis, and conducted damage assessment by exploiting the concept of multifractal theory. Liu (2008) established a mathematical model to simulate the disintegration of red bed soft rock according to the fractal concept by studying the particle size and fractal characteristics of soft rock disintegration, the development trend of the numerical simulation results is basically the same as that of the measured data, which shows that the collapse process of soft rock is a fractal. Sun, Xue \& Yin (2019) established a single fracture triaxial stress-seepage coupling model, and discussed the influence of three-dimensional stress and water pressure on the permeability coefficient of fractured rock mass. Under the condition of sandstone, Ding \& Liu ( 2018) studied the influence factors of the stress and deformation of the surrounding rock of the tunnel. Based on field and laboratory investigations, Erguler \& Shakoor (2009) recommended a new approaches and a durability classification system to assess disintegration characteristics of clay-bearing rocks. This system may be useful to practitioners for identifying the type and degree of disintegration that may occur on a freshly exposed rock face. Ali Saeed \& Zeynab (2018) estimated geomechanical parameters such as rock quality designation and uniaxial compressive strength of Azad dam by ordinary kriging as a geostatistical method, and presented specified distribution maps for each parameter. Maruvanchery \& Kim (2019) focused on the understanding of how microscale damages induced by water saturation affect mesoscale mechanical and fracture properties compared with oven dried 
specimens along three notch orientations-divider, arrester, and short transverse. Carulla Corominas \& Mavrouli (2017) analyzed the impact-induced rock mass fragmentation in a rockfall by comparing the in situ block size distribution of the rock mass detached from the cliff face and the resultant rockfall block size distribution of the rockfall fragments on the slope. Yagiz (2018) studied properties of rock including strength, dry and saturated densities, effective porosity and sonic wave velocity, and investigated the impact of those properties on the degradability of rocks. Gautam (2016) compared the laboratory slaking behavior of common clay-bearing rocks to their slaking behavior under natural climatic conditions observed during one year experimental study. Five-cycle slake durability tests were performed in the laboratory on five claystones, five mudstones, five siltstones, and five shales. It shows that the laboratory test underestimates the field durability for claystones, and overestimates it for siltstones.

The traditional rock disintegration is generally expressed by the rock disintegration resistance index, which is determined by the dry wet cycle test, however, this method can not fully understand the law of development and evolution of rock disintegration process, and can not quantitatively contact the relationship between rock expansion and disintegration. In this paper, the fractal model of slate disintegration is established, and the characteristics of slate disintegration under water are studied by using the correlation fractal dimension of fractal theory.

State of Art

\section{Fractal model of slate disintegration}

Fractal theory is a new branch of modern mathematics, which mainly studies some irregular curves and properties with self similarity, it has the characteristics of inversion irregular figure, self square fractal transformation and self affine fractal etc.

The macro fracture of rock is caused by the evolution of a large number of small fracture groups, and the small fracture is formed by the accumulation of even smaller fracture evolution, therefore, this kind of self similar behavior will inevitably lead to the fractal characteristics of rock fragmentation distribution. The disintegration and fracture of slate after water absorption is random, which is also a fractal. Therefore, it is feasible to study the law and mechanism of rock disintegration by means of fractal theory and mathematical analysis. Fractal is characterized by fractal dimension.

Generally, the fractal dimension mentioned by people is based on self similarity. For the fractal description of water collapse of slate, the correlation fractal dimension defined by the following equation can be used,

$$
D=-\lim _{\varepsilon \rightarrow 0} \frac{\ln N(\varepsilon)}{\ln \varepsilon} \quad \text { or } N(\varepsilon) \propto \varepsilon^{-D}
$$

Where,

$\varepsilon=$ the scale

$N(\varepsilon)=$ the measurement at this scale

$D=$ the fractal dimension value of research object

From the above equation, as long as a series of $\varepsilon$ and corresponding $N(\varepsilon)$ are measured, in the double logarithmic coordinate, the slope of $N(\varepsilon)-\varepsilon$ straight line is the fractional dimension $D$. Combined with rock mass disintegration, fractal dimension is obtained by quality. It are assumed that the total mass of clastic particles after slate disintegration is $M$, mesh size is $\varepsilon$, the mass of debris with diameter less than $\varepsilon$ is $M(\varepsilon)$. Passing a series of sieves with a mesh diameter of $\varepsilon$, a series of $M(\varepsilon)$ will be obtained. The size-frequency distribution of disintegrated fragments is,

$$
\frac{M(\varepsilon)}{M}=1-\exp \left[-\left(\frac{\varepsilon}{\sigma}\right)^{b}\right]
$$

When $\varepsilon / \sigma \leq 1$, the above formula can be simplified as,

$$
\frac{M(\varepsilon)}{M}=\left(\frac{\varepsilon}{\sigma}\right)^{b}=\sigma^{-b} \varepsilon^{b}
$$

Where, 
$M=$ total mass of clastic particles after slate disintegration, $g$

$M(\varepsilon)=$ mass of debris with diameter less than $\varepsilon, g$

$\sigma=$ maximum size of rock block

$b=$ distribution parameters of rock

$M(\varepsilon) / M=$ cumulative percentage content of crushed stone with diameter less than $\varepsilon$.

The derivation of Eqs. (1) and (3) respectively, we get,

$$
\begin{array}{ll}
d N \propto \varepsilon^{-D-1} d \varepsilon & \text { Equation (4) } \\
d M \propto \varepsilon^{b-1} d \varepsilon & \text { Equation (5) }
\end{array}
$$

Because of the relationship between mass and block size, that is, $\mathrm{M}$ is proportional to $R^{3}$, we get,

$$
d N \propto \varepsilon^{-3} d M \quad \text { Equation (6) }
$$

Through Eqs. (4)-(6), we get the relation equation between the fractal dimension $D$ and the rock distribution parameter $b$, and is,

$$
\begin{array}{ll}
D=3-b \quad \text { Equation (7) }
\end{array}
$$

Where, $\mathrm{b}$ is the slope value of the straight line in $\lg \varepsilon \sim \lg (M(\varepsilon) / M)$ coordinate.

As long as we make the straight line under the coordinate system in a certain state, then we can get the slope value of the line in this state, that is $b$, and then we can calculate the fractal dimension $D$. Through the indoor immersion disintegration test of soft rock, the mass distribution of each particle group of the disintegration can be obtained. Combined with mathematical statistics and regression knowledge, a series of fractal dimension values can be obtained in different soaking time periods, so that the fractal dimension of the disintegration of slate after encountering water changes with time.

Materials and methods

\section{Structural characteristics and composition of rock samples}

The test sample of slate comes from a deep water tunnel. According to the classification of engineering geology and petrology, the test sample belongs to clay type slate, which is black cryptocrystalline dense block with imperfect plate structure. The lithology is relatively soft, with black stripes. The rock component is mainly composed of cryptocrystalline clay minerals, containing more fine-grained carbonaceous and a small amount of mica and quartz and other debris, which is in a semi directional structure. Among them, cryptocrystalline clay is 40-50\%, organic carbon is less than $30 \%$, mica debris is more than $15 \%$, quartz debris is about $5 \%$. The behavior of the rock sample is shown in Figure1.

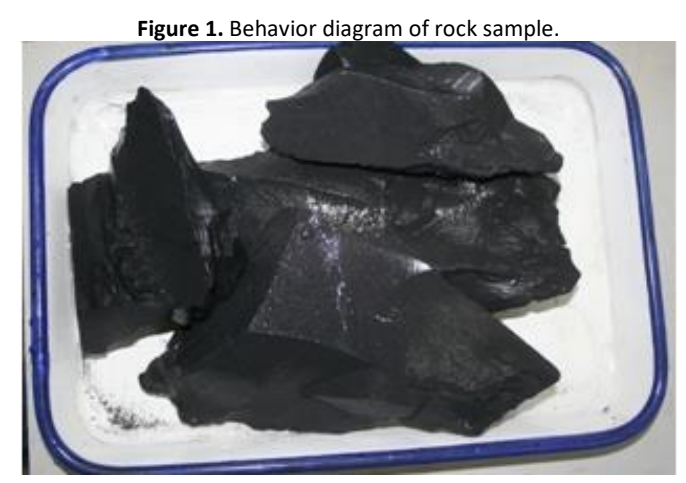

\section{Disintegration test}

In order to accurately reflect the field working conditions, clay slate with large volume and relatively complete structure is selected for test analysis. In order to obtain reliable data, the disintegration test is carried out as follows, 
1. Take slate in natural state, dip it into the water taken back from the site in a special container, and soak the rock sample.

2. The rock samples were soaked in water at intervals of $2 d, 6 d, 12 d, 17 d, 25 d, 38 d, 48 d$ and $75 d$ etc. After each stage, the rock block is put into the oven and baked for 12 hours under the constant temperature of $105^{\circ} \mathrm{C}$ according to the specification. Then, the rock blocks and disintegrated debris are screened by copper sieves of different specifications such as $20 \mathrm{~mm}, 10 \mathrm{~mm}, 5 \mathrm{~mm}, 2 \mathrm{~mm}, 0.5 \mathrm{~mm}, 0.25 \mathrm{~mm}, 0.075 \mathrm{~mm}$, and record the particle level. After screening, all levels of debris and rock blocks are mixed together for the next stage of water soaking.

3. According to the mathematical regression theory and fractal theory, the experimental data are sorted out. The fitting straight lines in $\lg \varepsilon \sim \lg (M(\varepsilon) / M)$ coordinate system of each soaking period are given respectively, and the slope of corresponding straight lines is calculated, and calculate the fractal dimension $D$, and establish the rule that the fractal dimension changes with time.

4. If the particle level changes little in a long time, the disintegration is basically stopped and the disintegration test is completed.

\section{Results and discussion}

After more than half a year of repeated soaking and drying disintegration tests, the test data related to the analysis of the disintegration characteristics of slate at the sampling point were obtained. Table 1 shows the total mass of rock samples under different soaking time and the particle size composition after sieving by copper screen with different apertures.

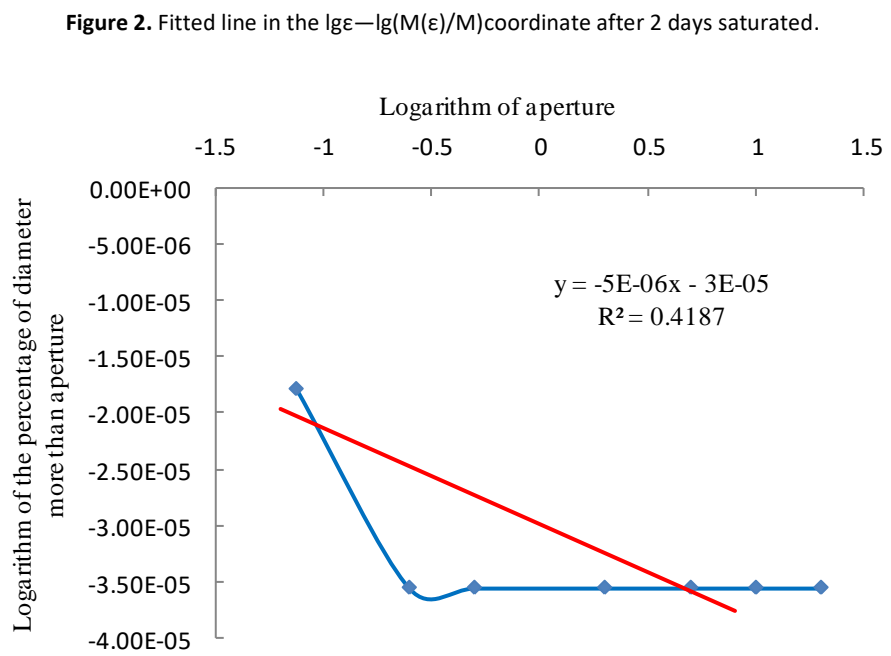

Table 1. Grade variety of slate samples during the collapsing test.

\begin{tabular}{cccccccc}
\hline $\begin{array}{c}\text { Water } \\
\text { soaking } \\
\text { time }\end{array}$ & $>0.075$ & $>0.25$ & $>0.5$ & $>2$ & $>5$ & $>10$ & $>20$ \\
\hline 2d & 99.9959 & 99.9918 & 99.9918 & 99.9918 & 99.9918 & 99.9918 & 99.9918 \\
6d & 99.9959 & 99.9918 & 99.9918 & 99.9918 & 99.9918 & 99.9918 & 99.9918 \\
$12 d$ & 99.9055 & 99.9014 & 99.8973 & 99.8973 & 99.8973 & 99.8973 & 99.8973 \\
$17 d$ & 99.9877 & 99.9836 & 99.9795 & 99.9754 & 99.9754 & 99.9754 & 99.9754 \\
25d & 99.9959 & 99.9918 & 99.9877 & 99.9836 & 99.9795 & 99.9795 & 99.9795 \\
38d & 99.9599 & 99.9877 & 99.9836 & 99.9795 & 99.9795 & 99.9795 & 99.9795 \\
48d & 99.9959 & 99.9877 & 99.9836 & 99.9815 & 99.9795 & 99.9795 & 99.9795 \\
$75 d$ & 99.9959 & 99.9877 & 99.9836 & 99.9815 & 99.9795 & 99.9795 & 99.9795 \\
100d & 99.9959 & 99.9877 & 99.9836 & 99.9815 & 99.9795 & 99.9795 & 99.9795 \\
\hline
\end{tabular}


According to the experimental results, the disintegration curve in $\lg \varepsilon-\lg (\mathrm{M}(\varepsilon) / \mathrm{M})$ coordinate after two days of water immersion is made, and the fitting line of the disintegration curve is made, so the slope of the fitting line is -0.000005 , as shown in Figure 2, and calculate the fractal dimension $D$ from Eq (7), D=3-b=3.000005. The disintegration curve and its fitting line under the disintegration coordinate system of rock sample after 100 days of water soaking are shown in Figure 3.

Figure 3. Fitted line in the $\lg \varepsilon-\lg (\mathrm{M}(\varepsilon) / \mathrm{M})$ coordinate after 100 days saturated.

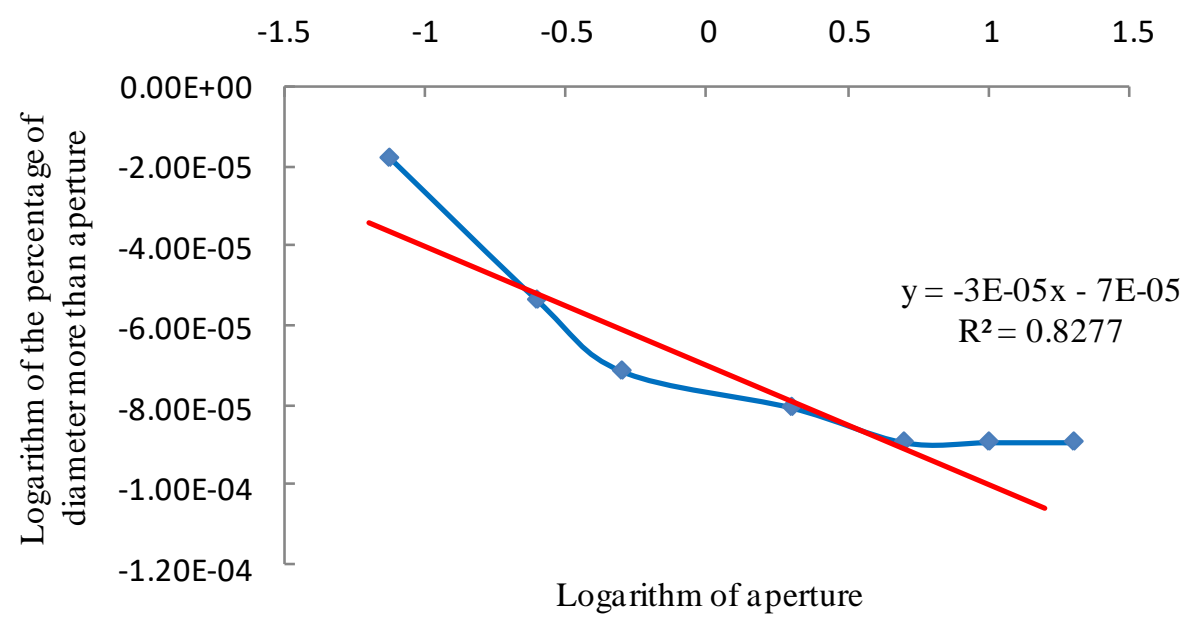

With the same method, the disintegration curve and fitting line with the soaking time of $6 d, 17 d, 25 d, 48 d$ and $75 d$ can be made, according to the slope of the fitting line, the corresponding fractal dimension value can be calculated by Equation 7. Table 2 is the calculation table of rock sample solution quality fractal dimension. According to Table 2, the curve of rock sample disintegration fractal dimension can be made, as shown in Figure 4, the law that the fractal dimension of slate disintegration changes with time is obtained.

Table 2. Calculation table of mass fractal dimension of rock sample disintegration.

\begin{tabular}{ccc}
\hline Time of water immersion/d & Slope of fitting line & Fractal dimension value \\
\hline $2 d$ & $-5.00 \mathrm{E}-06$ & 3.000005 \\
$6 \mathrm{~d}$ & $-5.00 \mathrm{E}-06$ & 3.000005 \\
$12 \mathrm{~d}$ & $-1.00 \mathrm{E}-05$ & 3.000010 \\
$17 \mathrm{~d}$ & $-2.00 \mathrm{E}-05$ & 3.000020 \\
$25 \mathrm{~d}$ & $-3.00 \mathrm{E}-05$ & 3.000030 \\
$38 \mathrm{~d}$ & $-3.00 \mathrm{E}-05$ & 3.000030 \\
$48 \mathrm{~d}$ & $-3.00 \mathrm{E}-05$ & 3.000030 \\
$75 \mathrm{~d}$ & $-3.00 \mathrm{E}-05$ & 3.000030 \\
$100 \mathrm{~d}$ & $-3.00 \mathrm{E}-05$ & 3.000030 \\
\hline
\end{tabular}

Figure 4. Qualitative fractal dimension variety with time during the samples collapsing test.

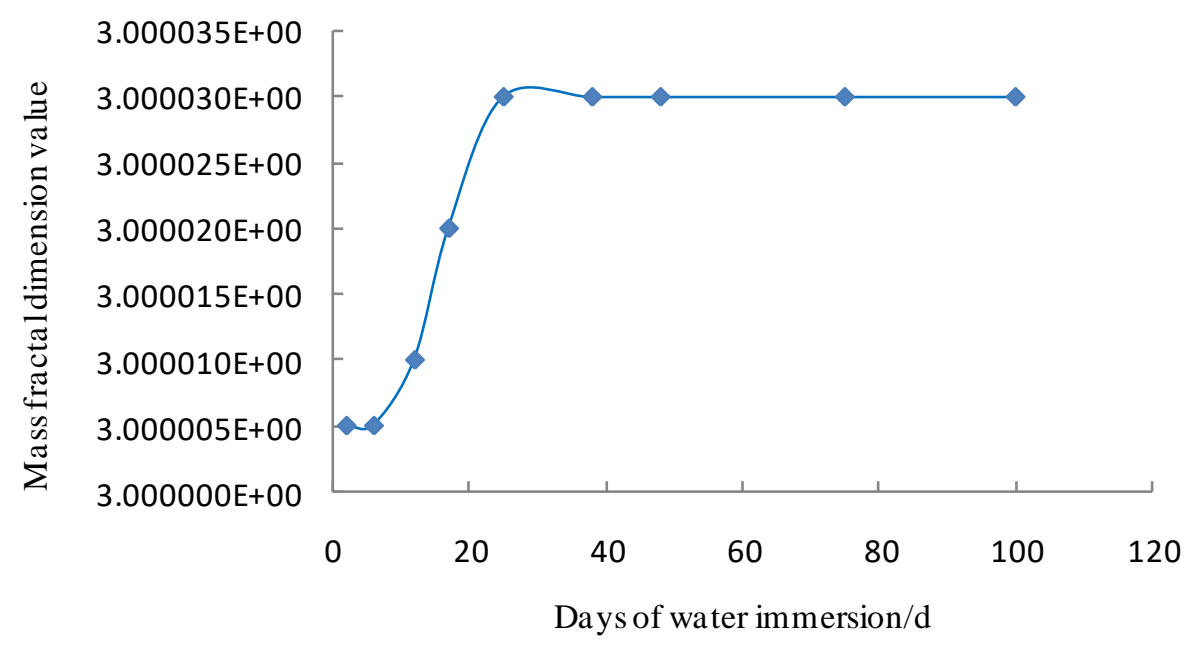


It can be seen from the curve of fractal dimension changing with time in Figure 4 and Table 2 that the disintegration of clay slate does not start when encountering water, there was no change in fractal dimension from the beginning of soaking to the 6th day, D is around 3.000005, it indicates that the disintegration degree is relatively low in this stage. During the period from 6 days to 17 days, the slope of the curve increases with time, that is, the fractal dimension increases with the increase of rock soaking time, and the rock shows strong disintegration characteristics. From 17 days to 25 days, the slope of fractal dimension curve decreased gradually, indicating that the degree of disintegration slightly decreased, but the curve is still rising, that is, the rock continues to disintegrate. It can also be seen from Table 1 that the percentage of particles larger than $0.25 \mathrm{~mm}$ and larger than $0.50 \mathrm{~mm}$ is still changing, which proves that disintegration is still continuing. Until the rock is soaked for more than 48 days, the fractal dimension and particle size composition will not change any more, indicating that the disintegration of rock has basically stopped.

For general sedimentary soft rock, the traditional disintegration test can reflect the disintegration property of rock, but it can not reflect the law that the disintegration characteristics of rock change with time. For slate and other rocks that have undergone metamorphism, the internal structure and composition of metamorphic rocks have changed a lot due to the influence of metamorphism such as high temperature and high pressure during the metamorphism of parent rock, which has changed the physical and hydraulic properties of rocks, therefore, the disintegration of rock has a lag phenomenon in time. Once disintegration occurs, it will last for a relatively long time.

Conclusions

It is found that the expansion and disintegration of slate is a multifractal process by tracking the change of particle size in the process of disintegration. In this paper, the mechanism of slate disintegration is analyzed by correlation fractal dimension, the fractal dimension of slate disintegration is solved by the method of quality fractal dimension, and the change of fractal dimension is used to reflect the characteristics of softening and disintegration of slate in water. The experimental results show that the fractal theory can be used to fully understand the development and evolution of rock disintegration process, which has a good guiding significance for engineering practice.

References

Ali, A., Saeed, S.M., \& Zeynab, R. (2018). Estimation of geomechanical parameters of tunnel route using geostatistical methods. Geomechanics and Engineering, 14(5), 453-458.

Bandis, S., Lumsden, A.C., \& Barton, N.K. (1981). Experimental studies of scale effects on the shear behaviour of rock joints. International Journal of Rock Mechanics and Mining Sciences \& Geomechanics Abstracts, 18(1), 1-21.

Carulla, R.R., Corominas. J., \& Mavrouli. O. (2017). A fractal fragmentation model for rockfalls. Landslides, 14(3), 875-889.

Ding, L.J., \& Liu, Y.H. (2018). Study on deformation law of surrounding rock of super long and deep buried sandstone tunnel. Geomechanics and Engineering, 16(1), 97-104.

Erguler, Z.A., \& Ulusay, R. (2009). Assessment of physical disintegration characteristics of clay-bearing rocks: Disintegration index test and a new durability classification chart. Engineering Geology, 105, 11-19.

Erguler, Z.A., \& Shakoor, A. (2009). Relative contribution of various climatic processes in disintegration of clay-bearing rocks. Engineering Geology, $108,36-42$.

Gautam, T.P., \& Shakoor, A. (2016). Comparing the Slaking of Clay-Bearing Rocks Under Laboratory Conditions to Slaking Under Natural Climatic Conditions. Rock Mech Rock Eng, 49, 19-31.

Lin, T.K., \& Chien, Y.H. (2017). A structural health monitoring system based on multifractal detrended cross-correlation analysis. Structural Engineering and Mechanics, 63(6), 751-760.

Liu, X.M., Zhao, M.H., \& Su, Y.H. (2008). Mathematical simulation of fractal mechanism for slaking of soft rock. Rock and Soil Mechanics, 29(8), 20432069.

Maruvanchery, V., \& Kim, E. (2019). Effects of water on rock fracture properties: Studies of mode I fracture toughness, crack propagation velocity, and consumed energy in calcite-cemented sandstone. Geomechanics and Engineering, 17(1), 57-67.

Sun, W.B., Xue, Y.C., \& Yin, L.M. (2019). Experimental study on seepage characteristics of large size rock specimens under three-dimensional stress. Geomechanics and Engineering, 18(6), 567-574.

Tating, F., Hack, R., \& Jetten, V. (2013). Engineering aspects and time effects of rapid deterioration of sandstone in the tropical environment of Sabah, Malaysia. Engineering Geology, 159, 20-30.

Vtorushin, E.V., \& Dorovsky, V.N. (2019). Application of non-stationary non-Euclidean model of inelastic deformations to rock cutting. Journal of Petroleum Science and Engineering, 177, 508-517.

Yagiz, S. (2018). The Effect of pH of the Testing Liquid on the Degradability of Carbonate Rocks. Geotech Geol Eng, 36, 2351-2363.

Zhu, X.H., Liu, W.J., \& Lv, Y.X. (2017). The investigation of rock cutting simulation based on discrete element method. Geomechanics and Engineering, 13(6), 977-995. 